\title{
Synthesis of Single Crystalline Tellurium Nanotubes with Triangular and Hexagonal Cross Sections
}

\author{
Paritosh Mohanty, Taejoon Kang, and Bongsoo Kim* \\ Department of Chemistry, KAIST, Daejeon 305-701, Korea \\ Jeunghee Park \\ Department of Chemistry, Korea University, Jochiwon 339-700, Korea
}

Received: September 12, 2005; In Final Form: November 16, 2005

\begin{abstract}
Single crystalline tellurium (Te) nanotubes with triangular cross sections were successfully synthesized for the first time by a simple approach of vaporizing tellurium metal and condensing the vapor in an inert atmosphere onto a suitable substrate. Tellurium gas was evaporated by heating at $350{ }^{\circ} \mathrm{C}$ and was condensed on the Si (100) substrate at $150-200{ }^{\circ} \mathrm{C}$, in the downstream of argon (Ar) gas at a flow rate of $25 \mathrm{sccm}$ for $10 \mathrm{~min}$. This led to the production of nanotubes of triangular cross section along with some hexagonal ones. The formation of the nanotubes was highly dependent upon the structure of the substrate surface, Ar gas flow rate, and the deposition temperature. When the substrate is $\mathrm{Si}$ (111) or sapphire (0001) or when the argon flow rate is increased to $500 \mathrm{sccm}$, nanowires and nanorods were exclusively formed. Irrespective of the morphologies, all the observed Te nanostructures grew in a regular [0001] direction. The facile approach to nanotubes with a triangular cross section may facilitate some new applications as well as stimulate theoretical studies pertaining to the stability of this high-energy configuration.
\end{abstract}

\section{Introduction}

Single crystalline, one-dimensional (1D) tubular nanostructures have attracted much interest because of their unique properties such as space-confined transport phenomena and potential applications such as fluid paths, reservoirs in catalysis, fuel cells, sensors, separation systems, and other novel applications in nanodevices. ${ }^{1-13}$ Much effort has been made in the past decade toward the study of carbon nanotubes with layered structures. ${ }^{1,2}$ Recently, processing of inorganic nanotubes with nonlayered structures received significant interest. Wu et al. synthesized aluminum nitride nanotubes by the reaction of aluminum powder in a $\mathrm{NH}_{3} / \mathrm{N}_{2}$ atmosphere in a horizontal tube furnace at a temperature of $1100{ }^{\circ} \mathrm{C} .{ }^{5} \mathrm{Li}$ et al. reported the synthesis of $\mathrm{In}_{2} \mathrm{O}_{3}$ nanotubes following a liquid metal assisted route. ${ }^{6}$ In some cases, nanowires were used as templates for the synthesis of single crystalline inorganic nanotubes. Yang and co-workers synthesized single crystalline GaN nanotubes using $\mathrm{ZnO}$ nanowires as templates. ${ }^{7} \mathrm{Hu}$ et al. employed $\mathrm{ZnS}$ nanowires as templates for the synthesis of $\mathrm{Si}$ nanotubes. ${ }^{8}$ Fabricating single crystalline nanotubes with controlled morphology is, however, still a challenge. Although it has been possible to precisely control the position, size, and crystallographic growth directions of a nanowire array utilizing proper characteristics of vapor-liquid-solid (VLS) growth mechanisms, ${ }^{14-20}$ control over the nanotube morphology has not been improved much. Synthesis of faceted hexagonal inorganic nanotubes have been reported for $\mathrm{AlN},{ }^{5} \mathrm{GaN},{ }^{7}$ and $\mathrm{ZnS} .{ }^{12} \mathrm{Hu}$ et al. synthesized single crystalline cubic GaN nanotubes with rectangular cross sections. ${ }^{10}$

Tellurium is a narrow-band gap semiconductor with a band gap energy of $0.35 \mathrm{eV}$. It has been considered to be an excellent

* Corresponding Author. E-mail: bongsoo@kaist.ac.kr. Fax: 82-42-8692810. Tel.: 82-42-869-2836. candidate for use in future high-efficiency photoconductors, thermoelectric, and piezoelectric devices..$^{3,21-32}$ Many functional materials can be easily synthesized by the reaction of tellurium with other elements. The crystal structure of $\mathrm{Te}$ is highly anisotropic with covalently bonded atoms forming unique helical chains. These chains are bound through weak van der Waals interactions and form hexagonal lattices. This anisotropic structure induces Te toward 1D growth. ${ }^{3,21-32}$ Mayer and Xia reported the synthesis of Te nanotubes with hexagonal cross sections by reducing orthotelluric acid in ethylene glycol. ${ }^{3}$ Wei et al. synthesized Te nanotubes by a solvothermal process using $N, N$-dimethylformamide as a solvent and porous silica (MCM41) as a growth assisting agent. ${ }^{23}$ Komarneni and co-workers demonstrated a biomolecule, alginic acid, assisted synthesis of Te nanowires. ${ }^{24}$ Zhu et al. reported the microwave assisted synthesis of Te nanorods and nanowires. ${ }^{25}$ Recently, He et al. demonstrated the growth of shuttlelike scrolled tellurium nanotubes and nanowires in a wet chemical method using amino acids as a growth assisting agent. ${ }^{26}$ Other methods for wet chemical synthesis of $1 \mathrm{D}$ tellurium nanostructures are also reported. ${ }^{27-29}$ However, the vapor phase synthesis of $\mathrm{Te}$ nanotubes is not common. In a particular case, it results in the formation of tubular structures of Te with micrometer diameters. ${ }^{30}$ Using a similar vaporization method, Geng et al. synthesized tellurium nanobelts by reacting $\mathrm{Al}_{2} \mathrm{Te}_{3}$ powder with $\mathrm{H}_{2} \mathrm{O}$ in a horizontal tube furnace at a temperature of $500{ }^{\circ} \mathrm{C} .{ }^{31}$ Métraux and Grobéty demonstrated the synthesis of Te nanotubes by a physical vapor deposition method using an induction furnace. ${ }^{32}$ Most of the reported Te nanotubes have hexagonal cross sections. To the best of our knowledge, so far, no report has demonstrated the synthesis of tubular nanostructures with triangular cross sections.

In this article, we report the synthesis of single crystalline tellurium nanotubes with triangular and hexagonal cross sections 
by a facile vaporization method. Most of the reported synthetic schemes for 1D tellurium nanostructures to date have used hydrazine, surfactant, biomolecules, other precursors, or some special experimental techniques. . $^{3,-32}$ The present method used only tellurium metal powder for the growth of the nanotubes. We will discuss the influence of deposition temperature, pressure, the flow rate of argon gas, and the substrates used for the synthesis on the morphology of the 1D tellurium nanostructures in this work. The fascinating electrical, electronic, or thermoelectric properties of Te together with the novel triangular morphology in the nanotube structure could potentially improve or modify its applications.

\section{Experimental Section}

Tellurium nanotubes with triangular and hexagonal cross sections were deposited on the Si (100) substrate placed in a 1 in. diameter horizontal quartz tube furnace, the central temperature of which was held at $350{ }^{\circ} \mathrm{C}$. The substrates were placed far downstream (about 3-4 in.) from an alumina boat filled with $0.3 \mathrm{~g}$ of tellurium powder (99.99\%, Aldrich). Argon flowed at a rate of $25 \mathrm{sccm}$ (standard cubic centimeter per minute), maintaining the chamber pressure at 1.0 to 1.5 Torr. The system was preevacuated to 0.3 Torr and then was purged with argon gas at a flow rate of $5 \mathrm{sccm}$ for $30 \mathrm{~min}$ and then at a rate of 25 sccm for another $30 \mathrm{~min}$. The high-temperature zone of the furnace was heated to $350{ }^{\circ} \mathrm{C}$ at a rate of $20{ }^{\circ} \mathrm{C} \mathrm{min}-1$. Grayishblack films were deposited all over the $\mathrm{Si}$ (100) substrate after a deposition time of $10 \mathrm{~min}$.

An X-ray diffraction (XRD) pattern of the specimen was recorded on a Rigaku D/max-rc $(12 \mathrm{~kW})$ diffractometer operated at $40 \mathrm{kV}$ voltage and $80 \mathrm{~mA}$ current with filtered $0.15405 \mathrm{~nm}$ $\mathrm{Cu}_{\mathrm{K} \alpha}$ radiation. Field emission scanning electron microscope (FESEM) images of Te nanotubes were taken on a Phillips XL30S. Transmission electron microscope (TEM) and highresolution TEM (HRTEM) images and selected area electron diffraction (SAED) patterns were taken on Philips TECNAIF20 and JEOL JEM-2100F transmission electron microscopes operated at $160 \mathrm{kV}$ and $200 \mathrm{kV}$, respectively. The sample for the TEM analysis was prepared by a standard procedure where the nanostructures are dispersed in the organic solvents and a drop of the solution is put on the carbon coated copper grid.

\section{Results and Discussion}

The simple method of thermal evaporation of Te metal and condensation of the vapor at a suitable temperature onto a substrate led to highly pure nanostructures in high yields. Figure $1 \mathrm{a}$ is the FESEM image showing an overview of the assynthesized tellurium nanotubes on a $\mathrm{Si}$ (100) substrate. Individual nanotubes with triangular and hexagonal cross sections are shown in Figures $1 \mathrm{~b}$ and c, respectively. As seen in the micrographs, the tubular nanostructures are straight and have open ends. They grow close to vertical to the substrate surface with some of the nanotubes inclined at an angle of 30$45^{\circ}$. The length of the triangular nanotube edges varies from 100 to $250 \mathrm{~nm}$, and the external diameter of the hexagonal nanotubes varies from 100 to $200 \mathrm{~nm}$. The lengths of both nanotubes are extended up to a few micrometers.

Figure 2a shows a typical XRD pattern of the obtained Te nanotubes. All the observed peaks in the diffractogram were successfully indexed assuming the hexagonal crystal structure of tellurium (JCPDS file no. 36-1452) with lattice parameters $a=0.44579 \mathrm{~nm}$ and $c=0.59270 \mathrm{~nm}$ of space group $P 3{ }_{1} 21$ (152). The peak positions are fairly matching with the standard

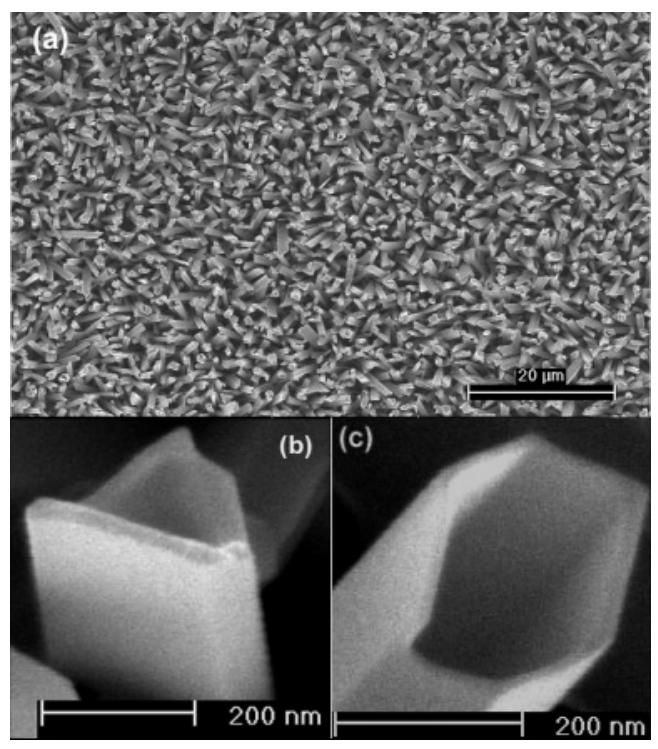

Figure 1. FESEM images of tellurium nanotubes synthesized on a $\mathrm{Si}$ (100) substrate: (a) overall view of the deposition of the nanotubes on the substrate; (b) individual nanotubes with triangular cross sections; (c) individual nanotubes with hexagonal cross sections.
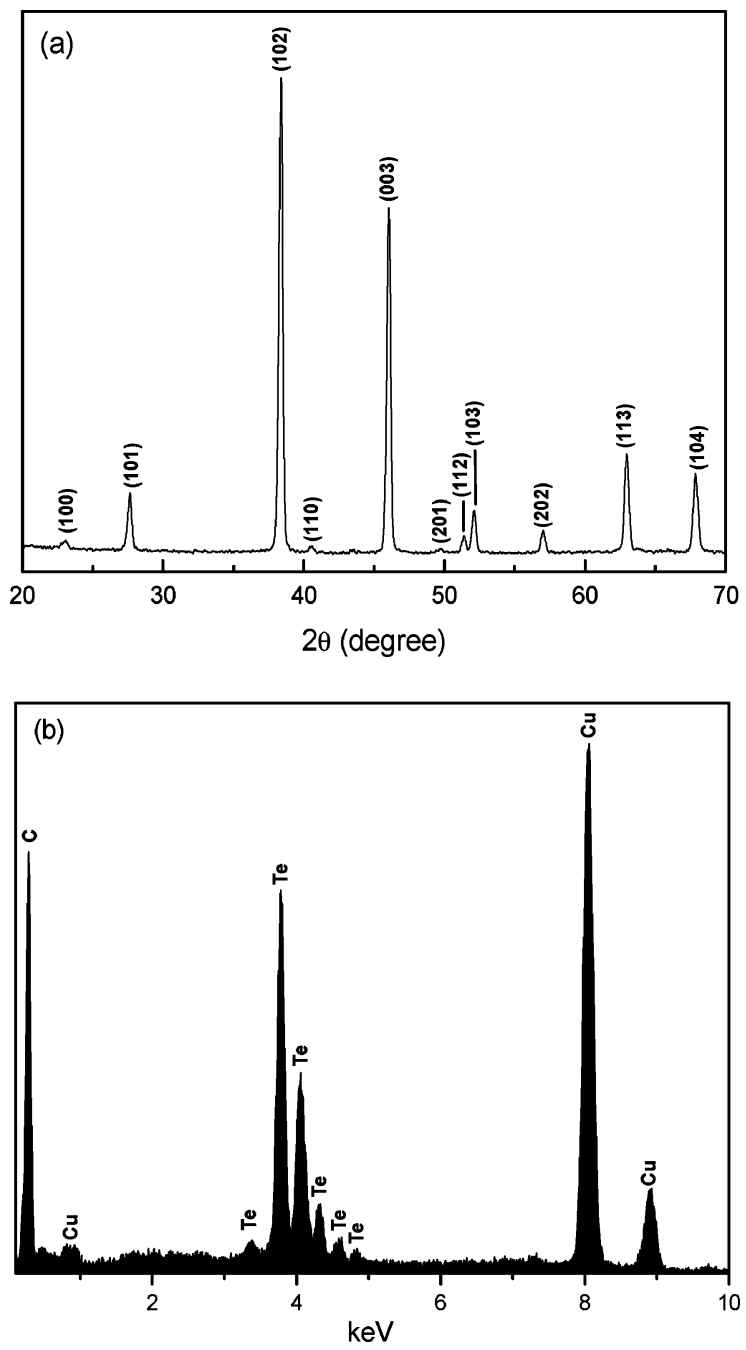

Figure 2. (a) XRD pattern and (b) EDS spectrum of Te nanotubes deposited on a Si (100) substrate by the thermal evaporation of $\mathrm{Te}$ metal at $350{ }^{\circ} \mathrm{C}$ for $10 \mathrm{~min}$.

values. The most intense peak is observed at interplanar spacing $d=0.2343 \mathrm{~nm}$ due to the reflection from the (102) crystal plane. 


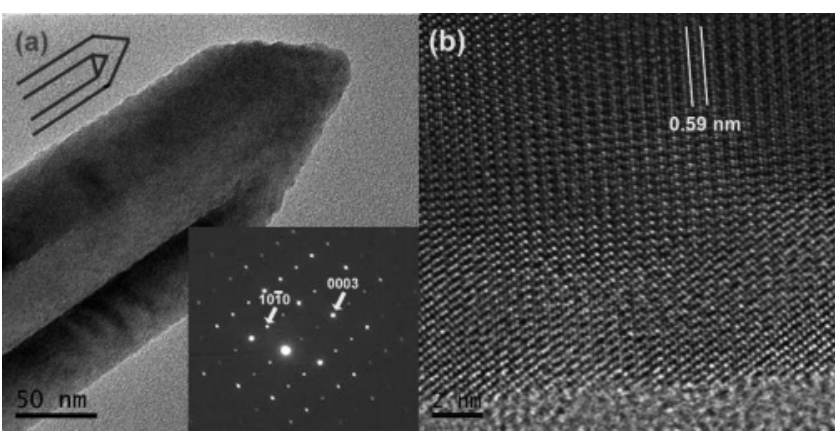

Figure 3. (a) TEM and (b) HRTEM micrographs showing the shape, morphology, and growth direction of a triangular cross section nanotube. The SAED pattern of the nanotube shown in the inset of part a was obtained by focusing the electron beam along the [010] zone axis.

The second most intense peak at $d=0.1969 \mathrm{~nm}$ is observed due to the reflection from the (003) crystal plane. In the standard diffraction file, the (003) peak is observed with an intensity of $\sim 9 \%$ with respect to the most intense peak. But in the present case, it appears as the second most intense peak with an intensity of $75 \%$ compared to the most intense (102) peak. This indicates that the nanotubes are single crystalline with a preferential [0001] growth direction. Since only tellurium peaks are seen in the energy-dispersive X-ray spectrum of Figure $2 b$ except some peaks from copper and carbon which are due to the use of a carbon coated copper grid for sample preparation, it is confirmed that the nanotubes contain only tellurium.

The HRTEM micrograph and the SAED pattern were used in order to confirm the structure and the growth direction of the as-synthesized nanotubes. Figure $3 \mathrm{a}$ and $\mathrm{b}$ displays TEM and HRTEM images of a representative triangular nanotube. The corresponding SAED pattern is given in the inset of Figure 3a. The TEM micrograph in Figure 3 a clearly shows the triangular morphology as well as the tubular structure of the nanotubes. The edge lengths of this triangular nanotube were calculated to be $\sim 130 \mathrm{~nm}$ with a wall thickness of $\sim 45 \mathrm{~nm}$. As can be seen with the HRTEM image in Figure 3b, the nanotube is structurally uniform and single crystalline. The interplanar spacing of $\sim 0.59 \mathrm{~nm}$, consistent with the (001) crystal plane, confirms that the nanotubes are grown in the preferred direction of [0001]. This is further supported by the SAED pattern in the inset of Figure 3a. It was reported that $1 \mathrm{D}$ Te nanostructures including nanowires and hexagonal nanotubes grew in the direction of [0001]..$^{3,21-32}$ Although the as-synthesized triangular nanotubes appear to be in a higher energy morphology than other 1D nanostructures, they still prefer to grow in the same [0001] direction.

The TEM and HRTEM images of a hexagonal nanotube are given in Figure $4 \mathrm{a}$ and $\mathrm{b}$, respectively. The external tube diameter shown in Figure $4 \mathrm{a}$ is $\sim 110 \mathrm{~nm}$. The SAED pattern (inset to Figure 4a) and the HRTEM image (Figure 4b) confirm that the hexagonal nanotubes are single crystalline in nature and have the same [0001] growth direction. In the TEM micrograph, five distinguishable contrast regions are seen. This and the FESEM image of Figure 1c confirm that the nanotube has a hexagonal cross section. A similar hexagonal cross section has been reported in $\mathrm{AlN},{ }^{5} \mathrm{GaN},{ }^{7}$ and $\mathrm{ZnS}^{12}$ nanotubes. It is reported that the hexagonal cross section $\mathrm{ZnS}$ nanotubes ${ }^{12}$ and the hexagonal cross section $\mathrm{GaN}$ nanowires ${ }^{17}$ grew along the [0001] direction and were closed by $\{1000\}$ side planes. Similarly, in the present case, the hexagonal nanotubes grew along the [0001] direction and were closed by $\{1000\}$ planes. The triangular cross section nanotubes, grown along the [0001]

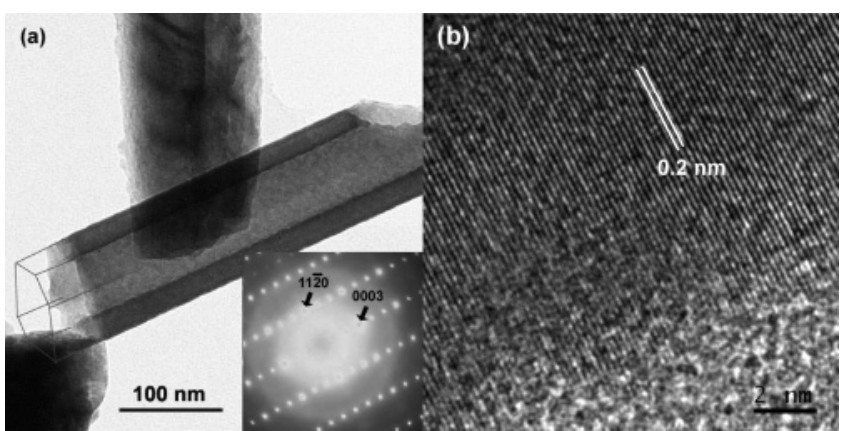

Figure 4. (a) TEM and (b) HRTEM images of a representative hexagonal cross section nanotube. The HRTEM image clearly shows interplanar spacing of $\sim 0.2 \mathrm{~nm}$ corresponding well with that of the [0003] lattice plane of hexagonal tellurium. The SAED pattern of the nanotube obtained by focusing the electron beam along the [110] zone axis is given in the inset part a, demonstrating the single crystalline nature of the specimen.

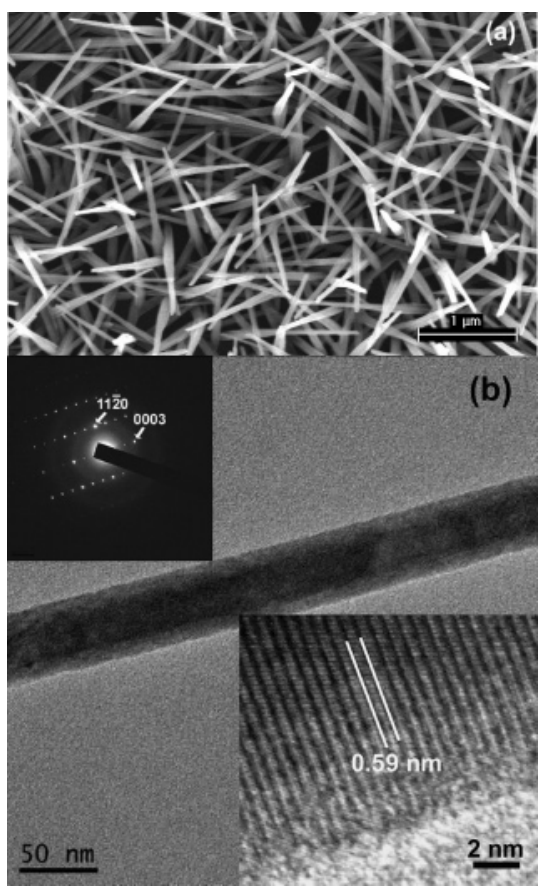

Figure 5. (a) FESEM image of tellurium nanowires deposited on a $\mathrm{Si}$ (100) substrate in a lower temperature zone $\left(<150{ }^{\circ} \mathrm{C}\right)$ of the quartz tube. Nanotubes were produced in a $150-200{ }^{\circ} \mathrm{C}$ temperature zone. (b) TEM image of a representative nanowire with a diameter of $\sim 50$ $\mathrm{nm}$. As demonstrated with the SAED pattern (top inset, electron beam was focused along [1ㅣㅣㄹ zone axis) and the HRTEM image (bottom inset), it is single crystalline with the preferred [0001] growth direction.

direction, were closed either by [1120], [ $\overline{2} 110]$, and [1 210$]$ planes or by [1 $\overline{1} 20]$, [21 $\overline{1} 0]$, and [ $\overline{1} 2 \overline{1} 0]$ planes. ${ }^{17}$

The triangular and hexagonal Te nanotubes were deposited on a substrate in the temperature range of $150-200{ }^{\circ} \mathrm{C}$. When the temperature is lower than $150^{\circ} \mathrm{C}, \mathrm{Te}$ nanowires were exclusively formed. A FESEM image of the as-synthesized Te nanowires is shown in Figure 5a. Unlike the nanotubes, thus-obtained nanowires did not grow vertical to the substrate surface, but they tended to lie along the substrate surface. They were deposited in large scale with their diameters varying from 30 to 60 $\mathrm{nm}$ and with lengths up to a few micrometers. The morphology of a representative nanowire can be further seen with the TEM image of the specimen shown in Figure 5b. The SAED pattern (top inset of Figure 5b) and HRTEM image (bottom inset of Figure $5 b$ ) of the nanowire show that the Te nanowires grew in the same [0001] direction as the nanotubes did. 


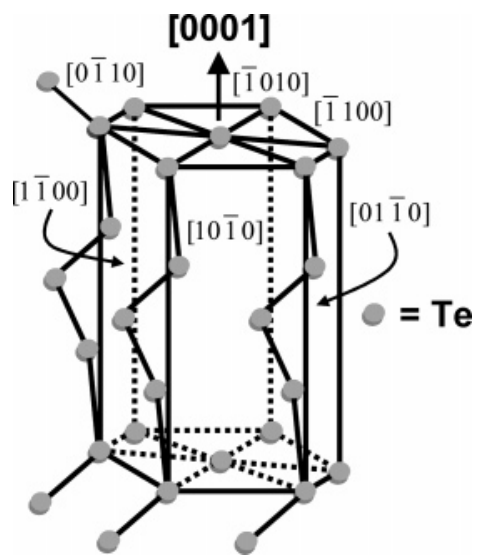

Figure 6. Schematic representation showing the hexagonal crystal structure of tellurium.

Wulff facets theorem for the shape-control of a crystal growth suggests that the relative specific surface energy of each crystal facet determined the shape of the crystal, and it favors the configuration with a minimum total surface energy. ${ }^{33}$ The symmetry of a basic crystal lattice is reflected in the shape of single-crystalline nanostructures. The crystal structure of Te as shown in Figure 6 is highly anisotropic with a longer $c$-axis and low-index [10 $\overline{1} 0],[01 \overline{1} 0],[1 \overline{1} 00],[0 \overline{1} 10],[\overline{1010}$, and [1100] crystallographic directions. It has covalently bonded atoms forming unique helical chains, which are bound through weak van der Waals interactions and form hexagonal lattices. Thus, this anisotropic crystal structure has a strong tendency toward 1D growth along the longer $c$-axis. . $^{3,21-32}$

However, the formation of the nanotubes and nanowires in different temperature regions can be explained as being similar to the mechanism proposed by Mayer and Xia for the wet chemical synthesis of the tellurium nanotubes and nanorods. ${ }^{3}$ It is well known that the degree of supersaturation plays an important role in the control over the morphology of the obtained nanostructures. In the synthesis of hexagonal Te nanotubes in the liquid phase, Mayer and Xia controlled the concentration of Te atoms by adjusting the concentration of orthotelluric acid, which produced Te atoms by the redox reaction with ethylene glycol. ${ }^{3}$ In our experiment, the degree of supersaturation is controlled by the temperature gradient in the reaction furnace. In the higher temperature region, if the degree of supersaturation is very slight, formation of many seeds on the substrate would greatly reduce the concentration of Te atoms near the substrate. As soon as the nucleation occurs, the additional tellurium atoms transported from the heated part of furnace will preferentially go to the circumferential edges because of the free-energy difference, facilitating the formation of the nanotubes. In the lower temperature region, the degree of supersaturation is higher than in the higher temperature region at the surface of substrate. It facilitates the nucleation of a large number of seeds, and also, the number of Te atoms available on the surface of a seed is larger with a reduced mobility of the Te atoms. This would favor the formation of nanowires which are lying down on the substrate.

When the Ar flow rate was increased from 25 to $500 \mathrm{sccm}$ and the pressure thus increased from 1 to 15 Torr with all other reaction conditions being kept same, only nanorods with similar triangular and hexagonal cross sections were deposited on the Si (100) substrate kept in the same temperature range of 150$200{ }^{\circ} \mathrm{C}$, as seen in the FESEM image (Figure 7). The dimension and growth direction of the nanorods are similar to those of the nanotubes. The nanorods tend to grow vertical to the substrate

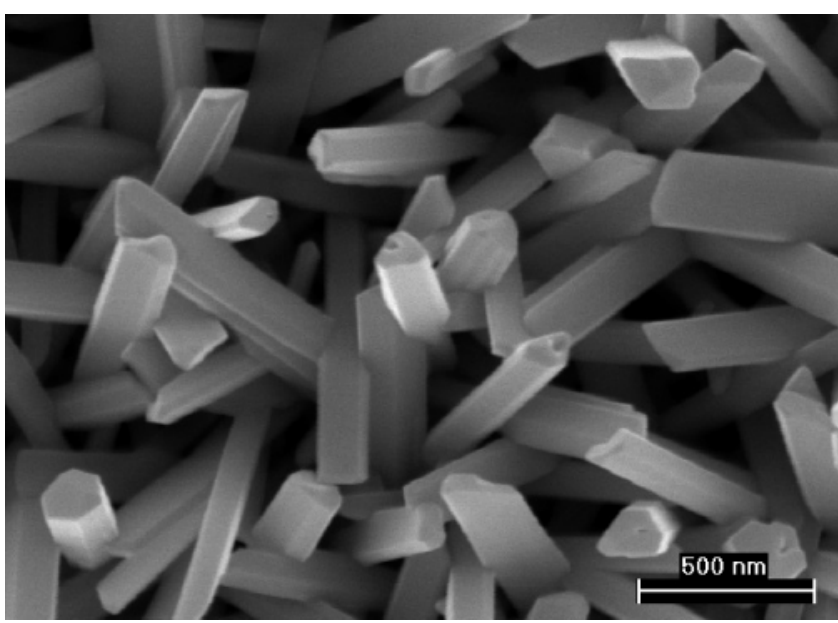

Figure 7. FESEM image of Te nanorods with triangular and hexagonal cross sections deposited on a $\mathrm{Si}(100)$ substrate. Only the nanorods were obtained when the Ar flow rate was increased from 25 to 500 sccm with all other reaction conditions being kept same, and no nanotube is produced.

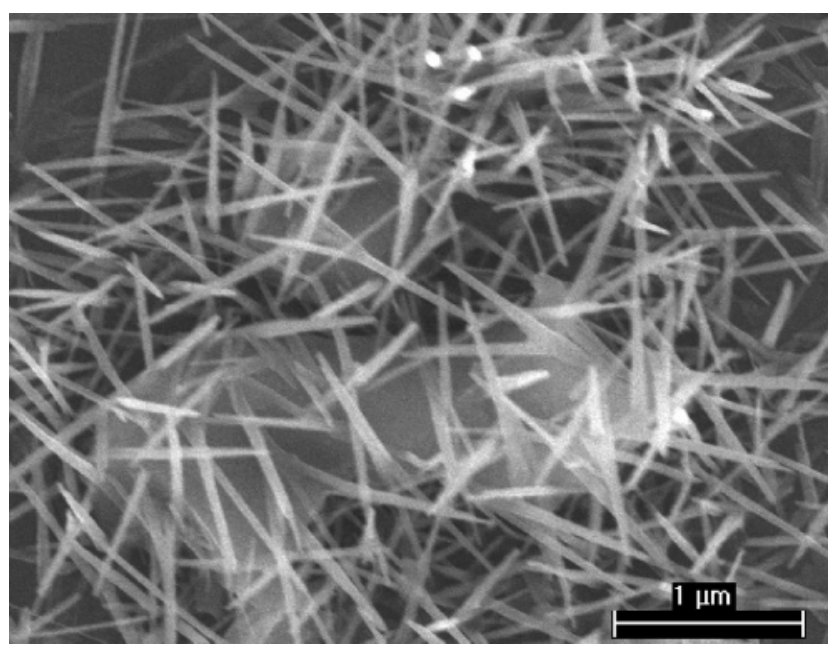

Figure 8. FESEM image of Te nanowires deposited on a Si (111) substrate at a reaction temperature of $350{ }^{\circ} \mathrm{C}$ with an $\mathrm{Ar}$ flow rate of $25 \mathrm{sccm}$ and at a pressure of 1 Torr. Similar microstructures were also observed when a sapphire (0001) substrate was used, and in both these cases, no nanotube was produced.

surface, with diameters and edge lengths comparable to the nanotubes. It is likely that the effect of collision by Ar atoms at high Ar flow rates dominates over the free energy factor and prevents a Te concentration profile from being formed on the seed. This would also favor the formation of nanorods or nanowires.

Recently, Yang and co-workers reported substrate-selective growth of $\mathrm{GaN}$ nanowires in triangular and hexagonal cross sections. ${ }^{17}$ When a (100) $\gamma-\mathrm{LiAlO}_{2}$ substrate was used, triangular cross section nanowires were grown along the [1ㅣㅣ direction perpendicular to the (100) crystal plane. Hexagonal cross section nanowires were obtained with the orthogonal [001] growth direction on a (111) $\mathrm{MgO}$ surface. In the present study, we found that both the triangular and hexagonal cross section nanotubes were grown in a regular [0001] direction on the $\mathrm{Si}$ (100) substrate. With this observation, it is not very clear whether there is a correlation between the substrate structure and the morphology of the synthesized Te nanotubes. Exclusive synthesis of either triangular or hexagonal cross section nanotubes on a substrate with a specific crystal orientation would not only reveal the clue to the growth mechanism but also be very 
important in the selective control of the nanostructures. Further study is required to accomplish this goal.

To find out more of the mechanism of nanotube formation, we repeated several batches of reactions on different substrates under similar experimental conditions, e.g., Si (111) and sapphire (0001). On both of the substrates, only nanowires with similar morphology were formed but no triangular or hexagonal nanotube was produced. This is shown with the FESEM image of the as-synthesized nanowires in Figure 8 deposited on the Si (111) substrate with all other reaction conditions being kept the same as for the Te nanotube synthesis on the $\mathrm{Si}$ (100) substrate (temperature $=350{ }^{\circ} \mathrm{C}$, Ar flow rate $=25 \mathrm{sccm}$, and pressure of 1 Torr). Detailed studies of the relationship between substrate structures and the morphology of the obtained nanostructures are under progress. Thus, appropriate substrates, deposition temperature ranges, and argon flow rates should be chosen in order to selectively synthesize the triangular and hexagonal nanotubes.

\section{Conclusions}

In summary, for the first time, we have successfully synthesized single crystalline tellurium nanotubes with a triangular cross section. Hexagonal cross section nanotubes were also formed at the same time. Free-standing nanotubes were grown in a regular [0001] direction on the $\mathrm{Si}(100)$ substrate surface at a deposition temperature of $150-200{ }^{\circ} \mathrm{C}$. Nanowires were exclusively obtained in the lower temperature region. The tellurium nanostructures were very pure as only Te metal was involved in the growth process and no template or catalyst was used. The obtained nanotubes with triangular cross sections can be potentially useful for the fabrication of nanodevices with high performance and novel properties. Further studies on the selective synthesis of the triangular and hexagonal nanotubes and electrical transport properties are under progress.

Acknowledgment. The authors thank the MOST for the support of "Center for Nanostructured Materials Technology" (05K1501-01210). SEM and TEM analysis was performed at the Korea Basic Science Institute in Daejeon. We thank Prof. S. Samal for his valuable discussions.

\section{References and Notes}

(1) Kong, J.; Soh, H. T.; Cassell, A. M.; Quate, C. F.; Dai, H. Nature 1998, 395, 878 .
(2) Schlittler, R. R.; Seo, J. W.; Gimzewski, J. K.; Durkan, C.; Saifullah, M. S. M.; Welland, M. E. Science 2001, 292, 1136.

(3) Mayer, B.; Xia, Y. Adv. Mater. 2002, 14, 279.

(4) Bakkers, E. P. A. M.; Verheijen, M. A. J. Am. Chem. Soc. 2003 125,3440 .

(5) Wu, Q.; Hu, Z.; Wang, X.; Lu, Y.; Chen, X.; Xu, H.; Chen, Y. J. Am. Chem. Soc. 2003, 125, 10176.

(6) Li, Y.; Bando, Y.; Golberg, D. Adv. Mater. 2003, 15, 581.

(7) Goldberger, J.; He, R.; Zhang, Y.; Lee, S.; Yan, H.; Choi, H.; Yang, P. Nature 2003, 422, 599.

(8) Hu, J.; Bando, Y.; Liu, Z.; Zhan, J.; Golberg, D.; Sekiguchi, T. Angew. Chem., Int. Ed. 2004, 43, 63.

(9) Krusin-Elbaum, L.; Newns, D. M.; Zeng, H.; Derycke, V.; Sun, J. Z.; Sandstrom, R. Nature 2004, 431, 672.

(10) Hu, J.; Bando, Y.; Zhang, J.; Xu, F.; Sekiguchi, T.; Golberg, D. Adv. Mater. 2004, 16, 1465.

(11) Liu, Z.; Zhang, D.; Han, S.; Li, C.; Lei, B.; Lu, W.; Fang, J.; Zhou, C. J. Am. Chem. Soc. 2005, 127, 6.

(12) Yin, L. W.; Bando, Y.; Zhan, J. H.; Li, M. S.; Golberg, D. Adv. Mater. 2005, 17, 1972.

(13) Fan, R.; Karnik, R.; Yue, M.; Li, D.; Majumdar, A.; Yang, P. Nano Lett. 2005, 5, 1633 .

(14) Wu, Y.; Yang, P. J. Am. Chem. Soc. 2001, 123, 3165.

(15) Xia, Y.; Yang, P.; Sun, Y.; Yu, Y.; Mayers, B.; Gates, B.; Yin, Y.; Kim, F.; Yan, H. Adv. Mater. 2003, 15, 353.

(16) Zhang, D.; Liu, Z.; Han, S.; Li, C.; Lei, B.; Stewart, M. P.; Tour, J. M.; Zhou, C. Nano Lett. 2004, 4, 2151.

(17) Kuykendall, T.; Pauzauskie, P. J.; Zhang, Y.; Goldberger, J.; Sirbuly, D.; Denlinger, J.; Yang, P. Nat. Mater. 2004, 3, 524.

(18) Guiton, B. S.; Gu, Q.; Prieto, A. L.; Gudiksen, M. S.; Park, H. J. Am. Chem. Soc. 2005, 127, 498.

(19) Gao, X. P.; Wang, Z. L. J. Phys. Chem. B 2004, 108, 7534.

(20) Wang, X.; Song, J.; Li, P. Ryou, J. H.; Dupuis, R. D.; Summers, C. J.; Wang Z. L. J. Am. Chem. Soc. 2005, 127, 7920.

(21) Mayer, B.; Xia, Y. J. Mater. Chem. 2002, 12, 1875.

(22) Mo, M.; Zeng, J.; Liu, X.; Yu, W.; Zhang, S.; Qian, Y. Adv. Mater. 2002, 14, 1658 .

(23) Wei, G.; Deng, Y.; Lin, Y. H.; Nan, C. W. Chem. Phys. Lett. 2003 , 372,590 .

(24) Lu, Q.; Gao, F.; Komarneni, S. Adv. Mater. 2004, 16, 1629.

(25) Zhu, Y. J.; Wang, W. W.; Qi, R. J.; Hu, X. L. Angew. Chem., Int. Ed. 2004, 43, 1410 .

(26) He, Z.; Yu, S. H.; Zhu, J. Chem. Mater. 2005, 17, 2785.

(27) Liu, Z.; Hu, Z.; Liang, J.; Li, S.; Yang, Y.; Peng, S.; Qian, Y. Langmuir 2004, 20, 214.

(28) Xi, G.; Peng, Y.; Yu, W.; Tian, Q. Cryst. Growth Des. 2005, 5, 325 .

(29) Tang, Z.; Wang, Y.; Sun, K.; Kotov, N. A. Adv. Mater. 2005, 17, 358.

(30) Li, X. L.; Cao, G. H.; Feng, C. M.; Li, Y. D. J. Mater. Chem. 2004, 14, 244.

(31) Geng, B.; Lin, Y.; Peng, X.; Meng, G.; Zhang, L. Nanotechnology 2003, 14, 983 .

(32) Metraux, C.; Grobety, B. J. Mater. Res. 2004, 19, 2159.

(33) Venables, J. A. Introduction to Surface and Thin Film Processes; Cambridge University Press: Cambridge, 2000; p 4. 